\title{
Number of Distinct 16-QAM Periodic Complementary Sequence Sets
}

\author{
Fanxin Zeng ${ }^{1,2}$ Xiaoping Zeng ${ }^{1}$ Zhenyu Zhang ${ }^{2}$ Guixin Xuan ${ }^{2}$ \\ 1 College of Communication Engineering, Chongqing University, Chongqing 400044, China \\ 2 Chongqing Key Lab. of Emergency Communication, Chongqing Communication Institute, Chongqing 400035, China \\ Email: \{fzengx,cqzhangzy\}@yahoo.com.cn, zxp@cqu.edu.cn
}

\begin{abstract}
Number of distinct sequences is an important parameter in sequence design due to the fact that such a parameter decides the number of on-line users of a communication system associating those sequences. In this paper, it is investigated that the number of distinct sequences from a construction, which was presented in IEEE WCSP'2012, producing a family of 16-QAM periodic complementary sequence mates. The investigation result shows that the aforementioned number equals the half value of length of sub-sequences in such mates.

Index Terms-complementary sequence set, distinct sequences, periodic correlation, 16-QAM constellation.
\end{abstract}

\section{INTRODUCTION}

To share all the properties: higher transmission data rates and spectrum efficiency, immunities to multiple access interference (MAI) and multi-path interference (MPI) synchronously, lower peak-to-mean envelope power ratio (PMEPR), and so forth, a communication system using QAM complementary sequences (CSs) is one of potential candidates. Recently, in this sense QAM complementary sequences are widely investigated. More clearly, it is investigated that the super bounds of PMEPR of several constructions of QAM sequences without CS pairs [1]-[4], whose results show that only QAM Golay CS pair results in the lowest super bound of PMEPR in an orthogonal frequency division multiplexing (OFDM) communication system. And 16-QAM and 64-QAM Golay CS pairs are constructed by [5]-[9]. As the development of investigation of QAM CS sequences, QAM CS pair is generalized to QAM CS set and QAM Golay CS pair is expanded to QAM periodic CS sets [10]-[13].

Based on quaternary periodic CS mates, a family of 16QAM periodic CS mates was presented in [14]. Since there are a large number of available quaternary periodic CS mates [15]-[17], the method in [14] can provide fairly rich 16-QAM periodic CS mates. In such a paper, however, the number of distinct such mates was not considered, which motivates our work to investigate it.

\section{PRELiminaries}

In this section, we will recall some basic concepts referred to in this paper, including a 16-QAM alphabet set, complementary sequences, and interleaved sequences.

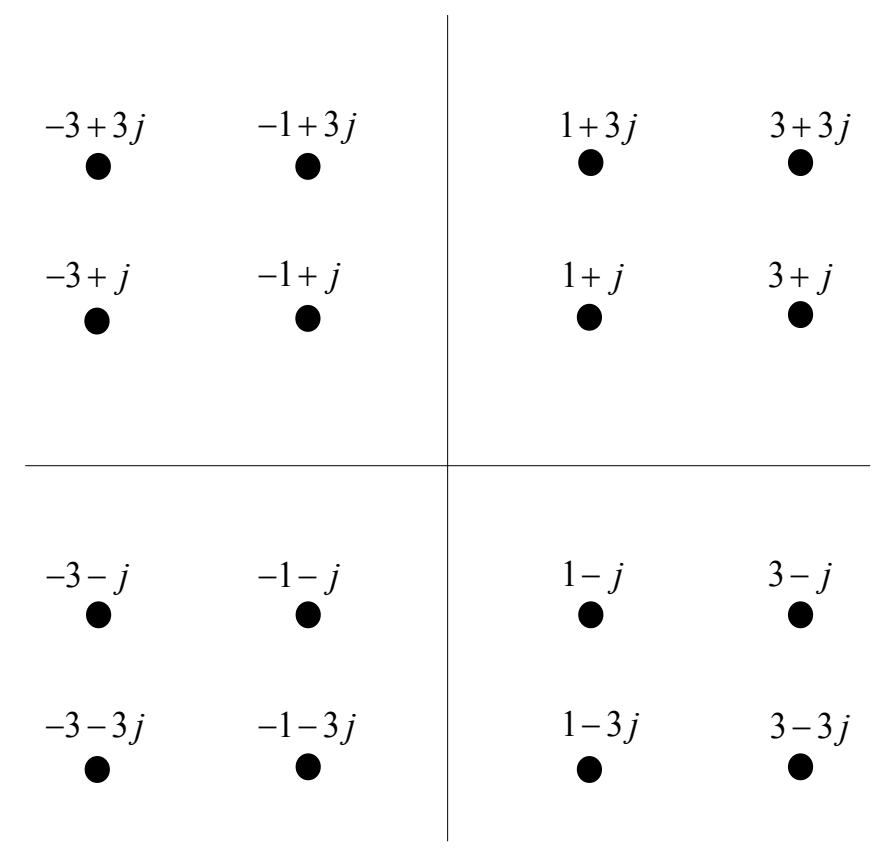

Fig. 1. 16-QAM constellation.

\section{A. 16-QAM Symbols}

A 16-QAM constellation is depicted in Fig. 1. In fact, the 16-QAM symbols can be driven by the quaternary phase shift keying (QPSK) symbols [3]-[4], which are given as follows.

$$
\begin{aligned}
& \left\{(1+j)\left(j^{a_{0}}+2 j^{a_{1}}\right) \mid a_{0}, a_{1} \in Z_{4}\right\}, \\
& \left\{(1-j)\left(j^{a_{0}}-2 j^{a_{1}}\right) \mid a_{0}, a_{1} \in Z_{4}\right\}, \\
& \left\{(1-j)\left(j^{a_{0}}+2 j^{a_{1}}\right) \mid a_{0}, a_{1} \in Z_{4}\right\}
\end{aligned}
$$

and

$$
\left\{(1+j)\left(j^{a_{0}}-2 j^{a_{1}}\right) \mid a_{0}, a_{1} \in Z_{4}\right\} .
$$

where $Z_{4}=\{0,1,2,3\}$, and the symbol $j$ denotes an imaginary unit, that is, $j^{2}=-1$.

\section{B. Complementary Sequences and interleaved Sequences}

Throughout this paper, let $U^{l}=\left(\underline{u}_{0}^{l}, \underline{u}_{1}^{l}, \cdots, \underline{u}_{M-1}^{l}\right)$ denote a sequence set that consists of $M$ sub-sequences, where $\underline{u}_{d}^{l}=$ $\left\{u_{d}^{l}(t)\right\}=\left(u_{d}^{l}(0), u_{d}^{l}(1), u_{d}^{l}(2), \cdots, u_{d}^{l}(N-1)\right)(0 \leq d \leq$ 
$M-1)$ denotes a complex sequence with length $N$, and $\overline{u_{d}^{l}(t)}$ represent the complex conjugate of $u_{d}^{l}(t)$.

Definition 1: For $\forall \underline{u}_{d}^{l} \in U^{l}$ and $\forall \underline{u}_{k}^{h} \in U^{h}$, for a time shift $\tau$, we refer to

$$
R_{u_{d}^{l}, u_{k}^{h}}(\tau)=\sum_{t=0}^{N-1} u_{d}^{l}(t) \overline{u_{k}^{h}(t+\tau)}
$$

as a periodic correlation function (CF) between $\underline{u}_{d}^{l}$ and $\underline{u}_{k}^{h}$, where the addition $t+\tau$ is calculated modulo $N$. If $l=h$ and $d=k, R_{u_{d}^{l}, u_{d}^{l}}(\tau)$ is called a periodic autocorrelation function (ACF), otherwise, a periodic crosscorrelation function (CCF).

Definition 2: Let $U^{l}=\left(\underline{u}_{0}^{l}, \underline{u}_{1}^{l}, \cdots, \underline{u}_{M-1}^{l}\right)$ consist of $M$ sub-sequences with each of length $N$. If we have

$$
\sum_{k=0}^{M-1} R_{u_{k}^{l}, u_{k}^{l}}(\tau)=\left\{\begin{array}{lll}
>0 & \tau \equiv 0 & (\bmod N) \\
0 & \tau \not \equiv 0 & (\bmod N),
\end{array}\right.
$$

we refer to the sequence set $U^{l}$ as a periodic CS set, denoted by $P C S S_{H}\left(U^{l}, M, N\right)$, where $H=4$ and $H=16$-QAM imply that the corresponding sequences are quaternary and 16-QAM ones, respectively. When $M=2$, the sequence set $U^{l}$ is called a periodic CS pair.

Definition 3: For $\operatorname{PCSS}_{H}\left(U^{l}, M, N\right)$ and $\operatorname{PCSS}_{H}\left(U^{h}\right.$, $M, N)$, if we have

$$
\sum_{k=0}^{M-1} R_{u_{k}^{l}, u_{k}^{h}}(\tau)=0 \quad(\forall \tau),
$$

we say that the sequence sets $U^{l}$ and $U^{h}$ are the mate to each other.

Definition 4: Let the symbol $L$ denote a left cyclic shift operator. More clearly, for a sequence $\underline{u}_{d}^{l} \in U^{l}$ and an integer $\zeta$

$$
L^{\zeta} \underline{u}_{d}^{l}=\left(u_{d}^{l}(\zeta), u_{d}^{l}(\zeta+1), \cdots, u_{d}^{l}(\zeta+N-1)\right),
$$

in which the addition $\zeta+t(t \in[0, N-1])$ is performed modulo $N$. For two sequences $\underline{u}_{d}^{l}$ and $\underline{u}_{k}^{h}$, if there exists an integer $\zeta$ so that

$$
\underline{u}_{d}^{l}=L^{\zeta} \underline{u}_{k}^{h},
$$

these two sequences are said to be equivalent, or else to be distinct. For $P C S S_{H}\left(U^{l}, M, N\right)$ and $P C S S_{H}\left(U^{h}, M, N\right)$, if there are an integer $\zeta$ so that

$$
\underline{u}_{k}^{l}=L^{\zeta} \underline{u}_{k}^{h} \quad(0 \leq k \leq M-1),
$$

these two periodic CS sets are said to be equivalent, otherwise, to be distinct.

Definition 5: For the sequences $\left\{u_{d}^{l}(t)\right\}$ and $\left\{u_{k}^{h}(t)\right\}$ with each of period $N$, we construct a new sequence, called an interleaved sequence and denoted by $\left\{u_{d}^{l}\left(t^{\prime}\right)\right\} \odot\left\{u_{k}^{h}\left(t^{\prime}\right)\right\}$ or $\underline{u}_{d}^{l} \odot \underline{u}_{k}^{h}$, as follows.

$\underline{u}_{d}^{l} \odot \underline{u}_{k}^{h}=$

$\left(u_{d}^{l}(0), u_{k}^{h}(0), u_{d}^{l}(1), u_{k}^{h}(1), \cdots, u_{d}^{l}(N-1), u_{k}^{h}(N-1)\right)$,

which implies that an interleaved sequence has a length $2 N$.

\section{KNOWN CONSTRUCTION FOR YIELDING 16-QAM Periodic Complementary Sequence Mates}

Consider the quadriphase periodic CS mates $P C S S_{4}\left(U^{l}\right.$, $M, N)$ and $P C S S_{4}\left(U^{h}, M, N\right)$. Hence, by making use of an interleaving technique, we construct two classes of 16-QAM sequences with length $2 N$ as follows [14].

$$
\underline{x}_{k}^{l, h}=\underline{v}_{k}^{+, l, h} \odot \underline{v}_{k}^{-, l, h}
$$

and

$$
\underline{y}_{k}^{l, h}=\underline{w}_{k}^{+, l, h} \odot \underline{w}_{k}^{-, l, h},
$$

where for $\forall k(0 \leq k \leq M-1)$, we have

$$
\begin{aligned}
& v_{k}^{+, l, h}(t)=(1+j)\left(j^{u_{k}^{l}(t)}+2 j^{u_{k}^{h}(t+\delta)}\right) \\
& v_{k}^{-, l, h}(t)=(1-j)\left(j^{u_{k}^{h}(t+\delta)}-2 j^{u_{k}^{l}(t)}\right) \\
& w_{k}^{+, l, h}(t)=(1-j)\left(j^{u_{k}^{l}(t)}+2 j^{u_{k}^{h}(t+\delta)}\right) \\
& w_{k}^{-, l, h}(t)=(1+j)\left(j^{u_{k}^{h}(t+\delta)}-2 j^{u_{k}^{l}(t)}\right),
\end{aligned}
$$

and $0 \leq \delta \leq N-1$.

We have the following conclusion.

Theorem 1 ([14]): The 16-QAM sequence sets $\left(X^{l, h}, M, 2 N\right)$ and $\left(Y^{l, h}, M, 2 N\right)$ are the mate to each other, where $X^{l, h}=\left(\underline{x}_{0}^{l, h}, \underline{x}_{1}^{l, h}, \cdots, \underline{x}_{M-1}^{l, h}\right)$ and $Y^{l, h}=\left(\underline{y}_{0}^{l, h}, \underline{y}_{1}^{l, h}, \cdots, \underline{y}_{M-1}^{l, h}\right)$.

\section{NEW RESUlts}

In this section, we will calculate the number of distinct sequences in the existing construction in [14].

Theorem 2: For quaternary periodic $\mathrm{CS}$ mate $P C S S_{4}\left(U^{l}, M, \quad N\right)$ and $P C S S_{4}\left(U^{h}, M, N\right)$, let two integers $\delta_{1}$ and $\delta_{2}$ satisfy $\delta_{1} \neq \delta_{2}\left(0 \leq \delta_{1}, \delta_{2} \leq N-1\right)$. And For $\forall k(0 \leq k \leq M-1)$ and $i=1,2$,

$$
\begin{aligned}
& v_{k, i}^{+, l, h}(t)=(1+j)\left(j^{u_{k}^{l}(t)}+2 j^{u_{k}^{h}\left(t+\delta_{i}\right)}\right) \\
& v_{k, i}^{-, l, h}(t)=(1-j)\left(j^{u_{k}^{h}\left(t+\delta_{i}\right)}-2 j^{u_{k}^{l}(t)}\right) .
\end{aligned}
$$

By applying to Eq. (12), we obtain two 16-QAM periodic CS sets $P C S S_{16-\mathrm{QAM}}\left(X_{1}^{l, h}, M, 2 N\right)$ and $P C S S_{16-\mathrm{QAM}}\left(X_{2}^{l, h}, M, 2 N\right)$, where

$\underline{x}_{k, 1}^{l, h}=\underline{v}_{k, 1}^{+, l, h} \odot \underline{v}_{k, 1}^{-, l, h}$ and $\underline{x}_{k, 2}^{l, h}=\underline{v}_{k, 2}^{+, l, h} \odot \underline{v}_{k, 2}^{-, l, h}$.

Then $P C S S_{16-\mathrm{QAM}}\left(X_{1}^{l, h}, M, 2 N\right)$ and $\operatorname{PCS} S_{16-\mathrm{QAM}}\left(X_{2}^{l, h}\right.$, $M, 2 N)$ are distinct. Further, By employing Eq.(12) $N$ distinct 16-QAM periodic CS sets are given.

Proof: Provided that for $\delta_{1} \neq \delta_{2}$, we have that

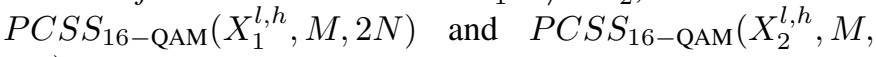
$2 N)$ are equivalent to each other, that is, there exists an integer $\zeta$ so as to satisfy $\underline{x}_{k, 1}^{l, h}=L^{\zeta} \underline{x}_{k, 2}^{l, h}(0 \leq k \leq M-1)$.Then, we proclaim that a contradictory must appear. In fact, for $\forall k$ we consider two cases: $\zeta=2 \pi$ and $\zeta=2 \pi+1(0 \leq \pi \leq N-1)$.

Case 1: $\zeta=2 \pi$.

Apparently, the relationship between the 16-QAM sequences $\underline{x}_{k, 1}^{l, h}$ and $L^{2 \pi} \underline{x}_{k, 2}^{l, h}$ is

$v_{k, 1}^{+, l, h}(0), v_{k, 1}^{-, l, h}(0), v_{k, 1}^{+, l, h}(1), \quad v_{k, 1}^{-, l, h}(1), \quad \ldots$
$v_{k, 2}^{+, l, h}(\pi), v_{k, 2}^{-, l, h}(\pi), v_{k, 2}^{+, l, h}(\pi+1), v_{k, 2}^{-, l, h}(\pi+1), \cdots$. 
As a consequence, from Definition 4 we have

$$
\left\{\begin{array}{l}
\underline{v}_{k, 1}^{+, l, h}=L^{\pi} \underline{v}_{k, 2}^{+, l, h} \\
\underline{v}_{k, 1}^{-, l, h}=L^{\pi} \underline{v}_{k, 2}^{-, l, h},
\end{array}\right.
$$

which reduces to

$$
\left\{\begin{array}{l}
j^{u_{k}^{l}(t)}-j^{u_{k}^{l}(t+\pi)}=2\left[j^{u_{k}^{h}\left(t+\delta_{2}+\pi\right)}-j^{u_{k}^{h}\left(t+\delta_{1}\right)}\right] \\
2\left[j^{u_{k}^{l}(t)}-j^{u_{k}^{l}(t+\pi)}\right]=j^{u_{k}^{h}\left(t+\delta_{1}\right)}-j^{u_{k}^{h}\left(t+\delta_{2}+\pi\right)},
\end{array}\right.
$$

where Eq.(15) is applied to. Further, Eq.(19) is simplified to

$$
\left\{\begin{array}{l}
j^{u_{k}^{l}(t)}=j^{u_{k}^{l}(t+\pi)} \forall t \\
j^{u_{k}^{h}\left(t+\delta_{2}+\pi\right)}=j^{u_{k}^{h}\left(t+\delta_{1}\right)} \forall t,
\end{array}\right.
$$

which implies both $\pi=0$ and $\delta_{1}=\delta_{2}$. Obviously, this is a contradictory.

Case 2: $\zeta=2 \pi+1$.

Similarly, the relationship between the 16-QAM sequences $\underline{x}_{k, 1}^{l, h}$ and $L^{2 \pi+1} \underline{x}_{k, 2}^{l, h}$ is

$v_{k, 1}^{+, l, h}(0), v_{k, 1}^{-, l, h}(0), \quad v_{k, 1}^{+, l, h}(1), \quad v_{k, 1}^{-, l, h}(1), \quad \cdots$
$v_{k, 2}^{-, l, h}(\pi), v_{k, 2}^{+, l, h}(\pi+1), v_{k, 2}^{-, l, h}(\pi+1), v_{k, 2}^{+, l, h}(\pi+2), \cdots$.

Hence, by Definition 4 we have

$$
\left\{\begin{array}{l}
\underline{v}_{k, 1}^{+, l, h}=L^{\pi} \underline{v}_{k, 2}^{-, l, h} \\
\underline{v}_{k, 1}^{-, l, h}=L^{\pi+1} \underline{v}_{k, 2}^{+, l, h},
\end{array}\right.
$$

After substituting Eq.(15) into Eq.(22), we have

$$
\left\{\begin{array}{c}
j^{u_{k}^{l}(t)}+2 j^{u_{k}^{h}\left(t+\delta_{1}\right)}= \\
-j\left[j^{u_{k}^{h}\left(t+\delta_{2}+\pi\right)}-2 j^{u_{k}^{l}(t+\pi)}\right] \\
j^{u_{k}^{h}\left(t+\delta_{1}\right)}-2 j^{u_{k}^{l}(t)}= \\
j\left[j^{u_{k}^{l}(t+\pi+1)}+2 j^{u_{k}^{h}\left(t+\delta_{2}+\pi+1\right)}\right] .
\end{array}\right.
$$

After multiplying $\overline{j^{l}(t)}$ from the right-hand in Eq.(23a), and applying to Eq.(5), we have

$$
\begin{aligned}
R_{u_{k}^{l}, u_{k}^{l}}(0)+ & 2 R_{u_{k}^{h}, u_{k}^{l}}\left(-\delta_{1}\right)= \\
& -j\left[R_{u_{k}^{h}, u_{k}^{l}}\left(-\delta_{2}-\pi\right)-2 R_{u_{k}^{l}, u_{k}^{l}}(-\pi)\right],
\end{aligned}
$$

which reduces to

$$
\sum_{k=0}^{M-1} R_{u_{k}^{l}, u_{k}^{l}}(0)=2 j \sum_{k=0}^{M-1} R_{u_{k}^{l}, u_{k}^{l}}(-\pi),
$$

due to Eqs.(6) and (7).

With the same argumentation, by multiplying $\overline{j^{u_{k}^{l}(t+1)}}$ from the right-hand in Eq.(23b) we have

$$
\begin{aligned}
R_{u_{k}^{h}, u_{k}^{l}}(1- & \left.\delta_{1}\right)-2 R_{u_{k}^{l}, u_{k}^{l}}(1)= \\
& j\left[R_{u_{k}^{l}, u_{k}^{l}}(-\pi)+2 R_{u_{k}^{h}, u_{k}^{l}}\left(-\delta_{2}-\pi\right)\right],
\end{aligned}
$$

which reduces to

$$
j \sum_{k=0}^{M-1} R_{u_{k}^{l}, u_{k}^{l}}(-\pi)=-2 \sum_{k=0}^{M-1} R_{u_{k}^{l}, u_{k}^{l}}(1)=0,
$$

due to Eqs.(6) and (7).
Hence, according to Eqs.(25) and (27) we have

$$
M N=\sum_{k=0}^{M-1} R_{u_{k}^{l}, u_{k}^{l}}(0)=0,
$$

which is apparently impossible.

In addition, when the parameter $\delta$ ranges over the range from 0 to $N-1$, the above derivation guarantees that $N$ resultant 16-QAM CS sets by Theorem 1 are distinct from one another.

Example 1: Consider the quadriphase periodic CS mate $\left\{\operatorname{PCSS}_{4}\left(U^{l}, 4,6\right) \mid l=1,2\right\}$, copied from [16].

$\left[\begin{array}{l}U^{1} \\ U^{2}\end{array}\right]=\left[\begin{array}{llll}\underline{u}_{0}^{1} & \underline{u}_{1}^{1} & \underline{u}_{2}^{1} & \underline{u}_{3}^{1} \\ \underline{u}_{0}^{2} & \underline{u}_{1}^{2} & \underline{u}_{2}^{2} & \underline{u}_{3}^{2}\end{array}\right]=\left[\begin{array}{llll}113002 & 203312 & 010101 & 300211 \\ 122033 & 331220 & 021130 & 232323\end{array}\right]$.

Then, when $\delta$ ranges over the range from 0 to 5 , by Theorem 2 the resultant six 16-QAM periodic CS mates are given in Table 1. It is not difficult for the reader to check their distinction from one another.

\section{CONClusion}

This paper derives the number of distinct 16-QAM complementary sequence sets so as to remedy the drawback in [14]. The proposed number suggests that the construction in [14] can produce $N$ distinct sequences, where $N$ equals the value of length of sub-sequences in the quaternary sequences employed.

\section{ACKNOWLEDGMENTS}

This work was supported by the National Natural Science Foundation of China (NSFC) under Grants 60872164, 61002034, 61171089 and 61271003, and the Ministry of Industry and Information Technology of China (No.Equipment[2010]307).

\section{REFERENCES}

[1] C. RöBing and V. Tarokh, "A construction of OFDM 16-QAM sequences having low peak powers," IEEE Trans. on Inf. Theory, vol.47, no.5, pp.2091-2094, July 2001.

[2] V. Tarokh and R. Sadjadpour, "Construction of OFDM M-QAM sequences with low peak-to-average power ratio," IEEE Trans. on Commun., vol.51, no.1, pp.25-28, Jan 2005.

[3] B. Tarokh and H. R. Sadjadpour, "Construction of OFDM-QAM sequences with low peak to average power ratio," IEEE Trans. Commun., vol.51, no.1, pp.25-28, Jan. 2003.

[4] H. R. Sadjadpour, "Construction of non-square M-QAM sequences with low PMEPR for OFDM systems," IEE Proc. Commun., vol.151, no.1, pp.20-24, Feb. 2004.

[5] C. Y. Chang, Y. Li, and J. Hirata, "New 64-QAM Golay complementary sequences," IEEE Trans. Inf. Theory, vol.56, no.5, pp.2479-2485, May 2010.

[6] H. Lee and S. H. Golomb, "A new construction of 64-QAM Golay complementary sequences," IEEE Trans. Inf. Theory, vol.52, no.4, pp.1663-1670, Apr. 2006.

[7] C. V. Chong, R. Venkataramani, and V. Tarokh, "A new construction of 16-QAM Golay complementary sequences," IEEE Trans. Inf. Theory, vol.49, no.11, pp.2953-2959, Nov. 2003.

[8] Y. Li, "Comment on "A new construction of 16-QAM Golay complementary sequences" and extension for 64-QAM Golay sequences," IEEE Trans. Inf. Theory, vol.54, no.7, pp.3246-3251, Jul. 2008.

[9] Y. Li, "A construction of general QAM Golay complementary sequences,"IEEE Trans. Inf. Theory, vol.56, no.11, pp.5765-5771, Nov. 2010. 
TABLE I

THE RESULTANT SIX 16-QAM PERIODIC CS MATES FROM THEOREM 2.

\begin{tabular}{|c|c|c|}
\hline$\delta$ & $X^{1,2}$ & $Y^{1,2}$ \\
\hline 0 & 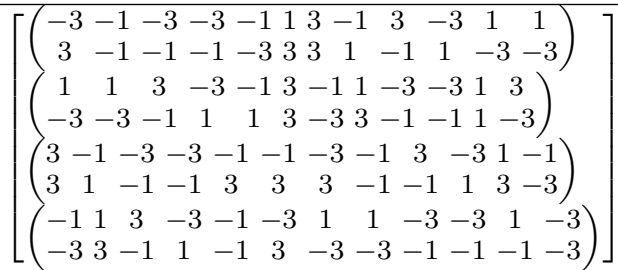 & 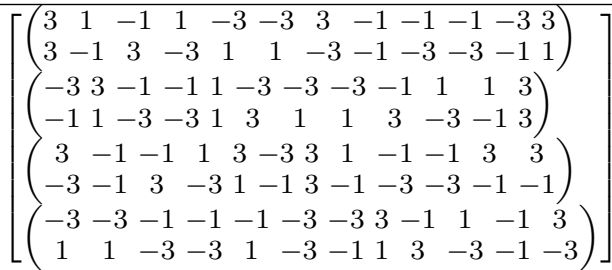 \\
\hline 1 & 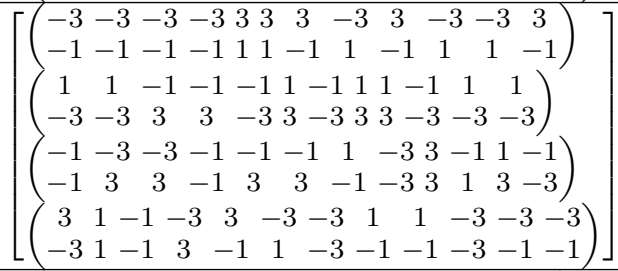 & 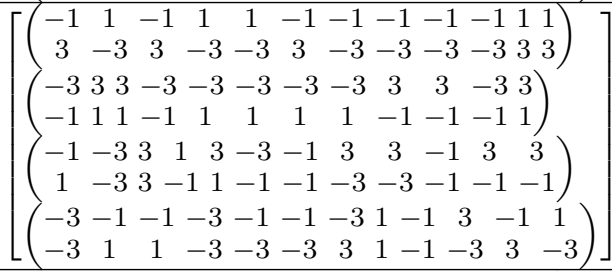 \\
\hline 2 & 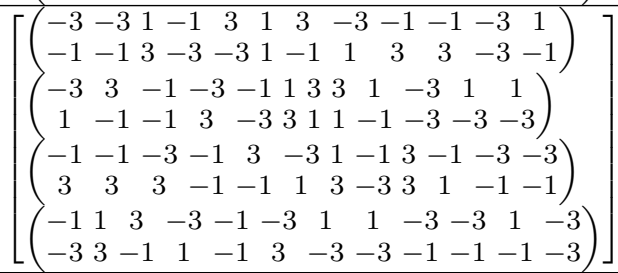 & 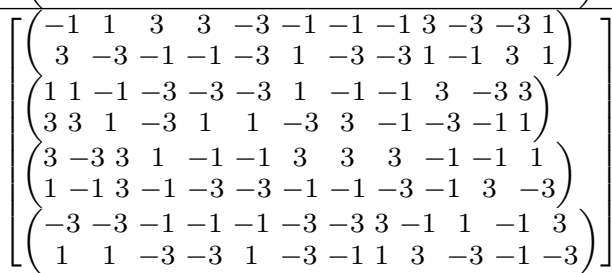 \\
\hline 3 & 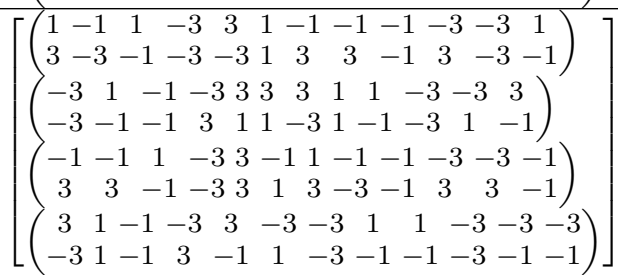 & 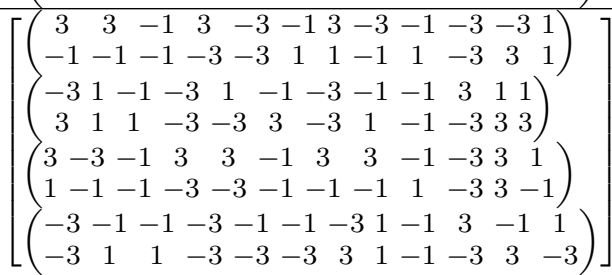 \\
\hline 4 & 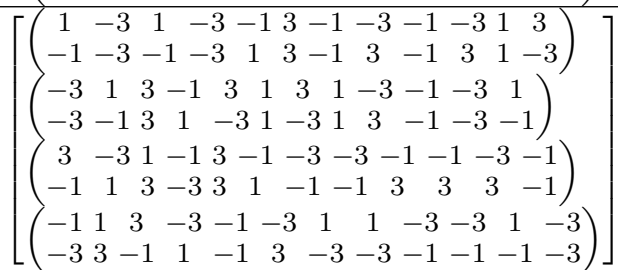 & 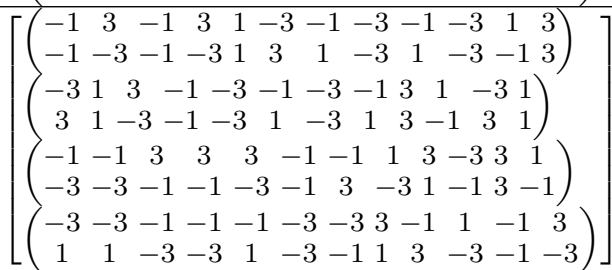 \\
\hline 5 & 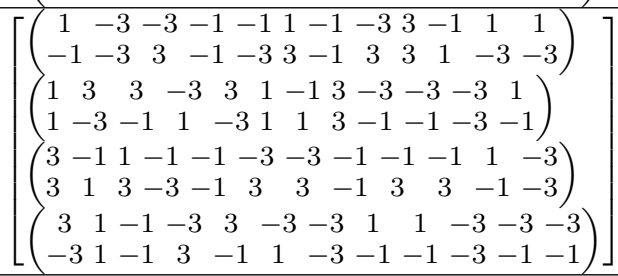 & 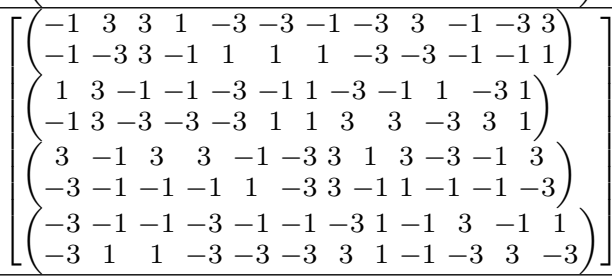 \\
\hline
\end{tabular}

[10] F. X. Zeng, X. P. Zeng, Z. Y. Zhang, and G. X. Xuan, "16-QAM Golay, Periodic and Z- Complementary Sequence Sets," IEICE Trans. Fundamentals, vol.E95-A, no.11, pp.2084-2089, Nov. 2012.

[11] F. X. Zeng, X. P. Zeng, Z. Y. Zhang, and G. X. Xuan, "Almost perfect sequences and periodic complementary sequence pairs over the 16-QAM constellation," IEICE Trans. Fundamentals, vol.E95-A, no.1, pp.400405, Jan. 2012.

[12] F. X. Zeng, X. P. Zeng, X. Y. Zeng, Z. Y. Zhang, and G. X. Xuan, "New Constructions of 16-QAM Periodic Complementary Sequences," IEEE Commun. Lett., vol.15, no.12, pp.2040-2043, Dec.2012.

[13] F. X. Zeng, X. P. Zeng, Z. Y. Zhang, and G. X. Xuan, " 8-QAM+ Periodic Complementary Sequence Sets," IEEE Commun. Lett., vol.16, no.1, pp.83-85, Jan. 2012.

[14] F. X. Zeng, X. P. Zeng, X. Y. Zeng, Z. Y. Zhang, and G. X. Xuan, "A Family of 16-QAM Periodic Complementary Sequence Mates for Suppressing Multiple Access Interference in CDMA Communication
Systems," Proc. of IEEE WCSP'2012, Huangshan, China, Oct.24-27, pp.1-5.

[15] J. W. Jang, Y. S. Kim, S. H. Kim, and D. W. Lim, "New construction methods of quaternary periodic complementary sequence sets," Advances in Mathematics of Communications, vol.4, no.1, pp.61-68, Feb. 2010.

[16] F. X. Zeng, X. P. Zeng, Z. Y. Zhang, and G. X. Xuan, "Quaternary periodic complementary/Z-complementary sequence sets based upon interleaving technique and Gray mapping," Advances in Mathematics of Communications, vol.6, no.2, pp.237-247, May 2012.

[17] F. X. Zeng, X. P. Zeng, Z. Y. Zhang, X. Y. Zeng, G. X. Xuan, and L. N. Xiao, "New construction method for quaternary aperiodic, periodic and Z- complementary sequence sets," Journal of Communications and Networks, vol14. no.3, pp.230-236, June 2012. 